\title{
RSSI Measurements of a GSM Signal within an Indoor Environment
}

\author{
Ahmad Fadzil Ismail ${ }^{1}$, Muhamad Jamil bin Jakpar ${ }^{1}$, Nor Farahidah Za'bah ${ }^{1}$, K. \\ Badron $^{1}$, and Wahidah Hashim ${ }^{2}$ \\ ${ }^{1}$ Kulliyyah of Engineering International Islamic University Malaysia, \\ ${ }^{2}$ College of Computer Science \& Info Tech, Universiti Tenaga Nasional Malaysia \\ muhamadjamiljakpar@gmail.com,af_ismail@iium.edu.my, \\ adah510@iium.edu.my,khairayu@iium.edu.my, andwahidah@uniten.edu.my
}

\begin{abstract}
Studies has suggested that the association between Received Signal Strength Indication (RSSI) and measured distance between the transmitter and receiver can be one of the key elements for ranging and localization technologies. In this study, measurements of RSSI and the distance between $1800 \mathrm{MHz}$ receiver and transmitter within an indoor environment were acquired, recorded and analyzed. Using the data, correlation for a select indoor environment was derived. By comparing the recorded distance values acquired at set RSSI with values calculated based on the Free Space Path Loss equation, provisional estimation of the indoor signal fading can be established. The experiment was the initial part of a three-stage work that will later entail the comparison to that of outdoor as well as clean room environments. The final result of the study hopes to achieve a simple yet improved indoor fading model experienced by mobile communication signals.
\end{abstract}

Keywords: RSSI, GSM1800, Indoor fading

\section{Introduction}

In the attempt to improve ranging and positioning technologies by means of Radio Frequency (RF) exploitation, it has become more critical to identify mathematical equations which can precisely link the association between the RSSI [1] readings and distance travelled by the signal. Received Signal Strength Indication (RSSI) is basically the received power at the receiver. It includes propagation loss due to distance travelled by the signal, the gain at the antenna/s as well as the loss. The RSSI analysis was made possible with the use of GSM radio transceiver units, a signal generator and a spectrum analyzer. In this paper, an association which can link the RSSI readings and measured distance within a specific indoor environment was derived and evaluated. The article is structured as follows: Section 2 - outlines on the theoretical steps in order to obtain the estimation model. Section 3 highlights the result and discussion of the conducted experiment. Section 4 points out the conclusion of the paper and the direction of future works.

\section{Variation of RSSI in a Specific Indoor Environment}

RSSI, Angle of Arrival (AOA) and Time Difference of Arrival (TDOA), are ranging technologies that are currently gaining popularity [4]. With the use of these technologies, ranging capability now can involve reduced implementation complexity, reduced overhead, and cheaper expenditure. It can be very suitable for portable receivers which have limited power source. RSSI can be described as the voltage output of the receiver which measured by a receiver's circuitry. Basically, it is more commonly to be measured 
as power (the squared magnitude of the signal strength). This removes the requirement for extra hardware in the receiver in order to measure the power of signal strength thus reduced the consumption of the power supply, size and cost. From reliable models of an RF signal propagation within a structure or other environments, RSSI could be exploited to produce dependable expanse estimation between the transmitter and the receiver. This approach necessitates detailed and proven models of RF propagation and comprises of all possible variants in receiver orientation and sensitivity [3].

\subsection{Principle of RSSI Ranging}

The theory of RSSI ranging involves the association between received power and transmitted power of wireless signals and the gap between the transmitter and receiver. This relationship is shown in (1).$P_{r}$ is the received power at the receiver. $P_{t}$ is the wireless signal's transmitted power. $d$ is the space between the transmitter and receiver. $n$ is the parameter which quantity depends on the propagation environment.

$$
P_{r}=P_{t} \times\left(\frac{1}{d}\right)^{n}[8]
$$

Equation (1) can be simplified into Equation (2) by multiplying 10 to the logarithm of both sides on (1),

$$
10 \log _{10} P_{r}=10 \log _{10} P_{t}-10 n \log _{10} d
$$

$P_{t}$, the transmitted power, and can be expressed in term of power unit of $\mathrm{dBm}$. Equation (2) can be rephrase as Equation (3).

$$
P_{r}(d B m)=A-10 n \log _{10} d
$$

It can be observed that the quantities of parameter $n$ and parameter $A$ are governed by Equation (3) outlining the association between the received signals strength and the spread how far the signal transmission had travelled.

\subsection{RSSI-based Ranging Equations}

Free-space model is one of the familiar model to determine the Path Loss. It is only valid to the following occasions: 1) the carrier wavelength $\lambda$ and the antenna size is much smaller than the transmission distance; 2) there is no obstruction between the receivers and the transmitters. With presumption that $P_{t}$ is the wireless signal's transmission power; the power of received signals, $P_{r}$ of receiver located within the span of $d$ can be ascertained using the ensuing formulas:

$$
\begin{aligned}
& P_{r}(d)=\frac{P_{t} G_{t} G_{r} \lambda^{2}}{(4 \pi)^{2} d^{2} L} \\
& P L(d B)=10 \log \frac{P_{t}}{P_{r}}=-10 \log \left[\frac{\lambda^{2}}{(4 \pi)^{2} d^{2}}\right]
\end{aligned}
$$

In (4), $\mathrm{L}$ is system loss factor where $G_{t}$ and $G_{r}$ are antenna gain. In most cases, values of $G_{t}=1, G_{r}=1$, and $L=1$ are commonly adopted. Equation (5) is the signal attenuation formula expressed in logarithmic scale. In Equation (5), the distance and the received power are 2-th power attenuation [3].

\subsection{Empirical Model for RSSI Ranging}

By knowing transmit power and received signal strength, the distance separation can be estimated. Using both values the propagation loss can also be computed. Empirical and theoretical and results of the calculation can be used for distance estimation. This method is widely deployed particularly for Radio Frequency signals. All signal power received are theoretically inversed proportion to the distance.

$$
\begin{aligned}
& P_{r} \propto \frac{1}{D^{n}} \\
& R S S \propto 10 \times \log \left(\frac{1}{D}\right)^{n}
\end{aligned}
$$




$$
R S S=-10 \times n \times \log (D)+C
$$

where $n$ is the path-loss exponent factor and $D$ is the separation gap between the receiver and the transmitter. $C$ can be deemed as a fixed constant. RSSI can be formulated as:

$R S S I=-m \times \log (D)+C$

For equation (11), $m$ represents the linear equation's slope between RSSI and $\log (\mathrm{D})$ is the path loss exponent factor summed up together as:

$$
n=m / 10
$$

\section{$2.4 \quad$ Indoor Experimental Set Up}

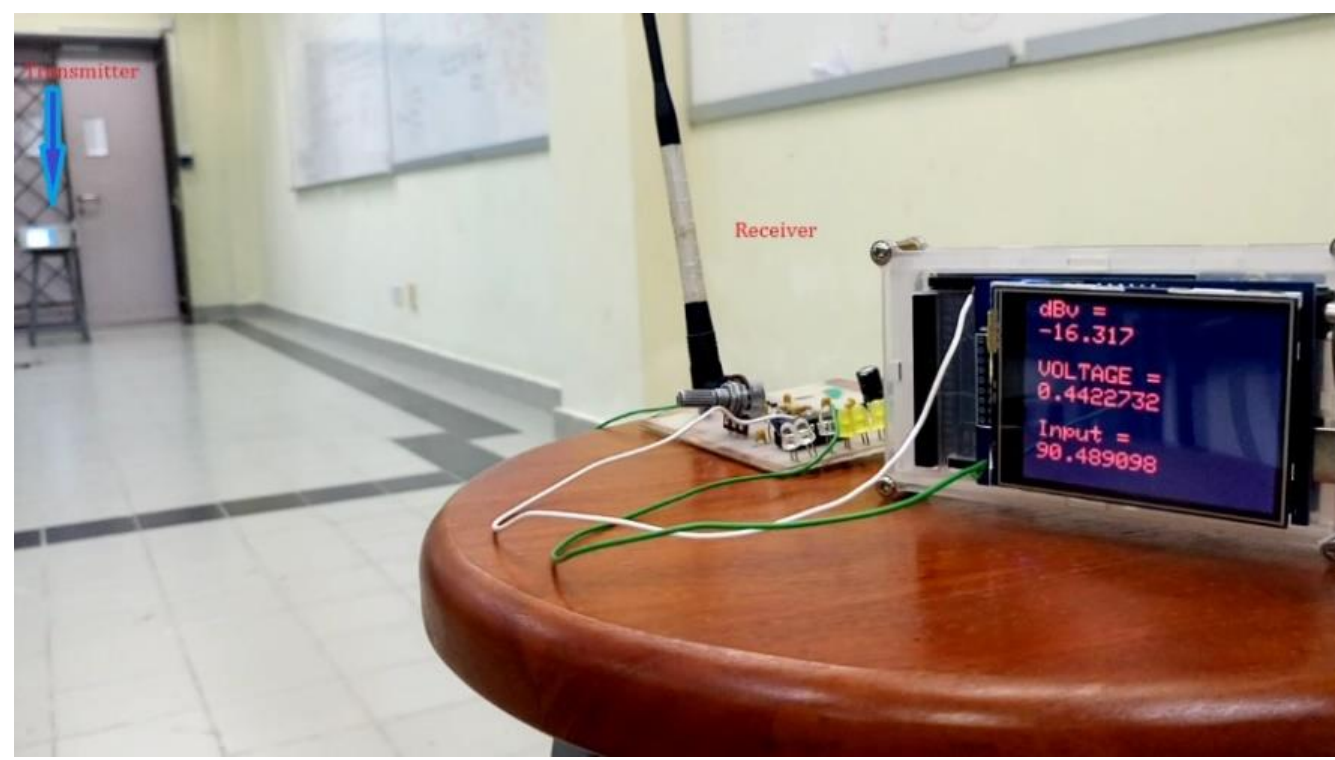

Figure 1. Experimental Set up

The experiment was a preliminary step in defining a measurement model estimation to define a distance based on RSSI. Figure 1 shows the experimental set up. The receiver that was used is a lab-assembled receiver founded on AD8313 IC chip. The circuit designed is able to detect signal in the range between $1.7 \mathrm{GHz}$ and $1.9 \mathrm{GHz}$. The $1.8 \mathrm{GHz}$ signal was selected for recording during the measurement campaign. The output of the receiver is the voltage value that can be consider as RSSI. Received power value in $\mathrm{dBm}$ is determined by connecting the receiver to the signal generator directly and adjusted to the available measured voltage. In hope to acquire the best result, the following precautions were adhere to. In every setup, the transmitter and receiver was arranged to be at the same height. Voltage supply to the receiver was fixed to $7.32 \mathrm{~V}$. There were no obstacle between the receiver and transmitter in order to ensure there was a clear line of sight between them. The antennas that were used at the receiver and transmitter were isotropic type. Signal generator was used instead of mobile phone since a signal generator is capable to provide a more stable power transmit. The receiver sensitivity was set to $43 \mathrm{dBm}$ as such would be able to avoid interference with transmitted signals by base stations. Receive signals power from the base stations in the areas selected identified using a spectrum analyzer is below $-45 \mathrm{dBm}$. In the attempt to avoid interference with uplink GSM signals from mobile phone call-out, no active phone device active was allowed at the testing area. The area involved in the testing is depicted in Figure 2. 


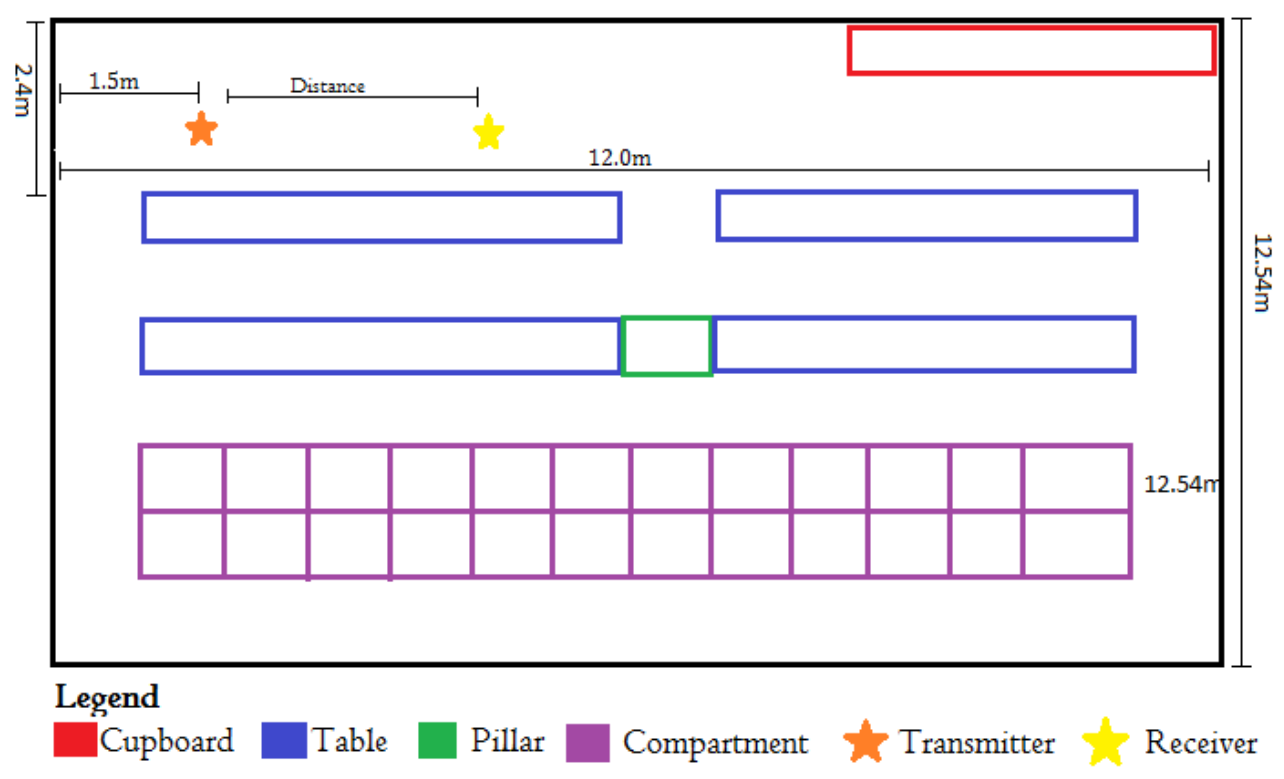

Figure 2. Indoor Environment Map

\section{Result and Discussion}

Distance can be estimated by using FSPL equation. As the distance between the transmitter and receiver increases, the value of the path loss will be higher. As path loss increases, RSSI decreases.



Figure 3. Calculated RSSI based on FSPL Model

From equation (5) the RSSI was calculated for distances between $1 \mathrm{~m}$ and $8 \mathrm{~m}$. Figure 3 shows the plot of calculated RSSI values at the receiver originated form transmitter with $20 \mathrm{dBm}$ power.

\subsection{Experimentation Analyses within an Enclosed Environment}

The outcome of the enclosed experimentation is presented in Figure 4. RSSI readings were then recorded at 1 meter interval distance up to 8 meter. Readings for every 
measurement were repeated 8 times within duration of 30 seconds. The plot of RSSI quantities in $\mathrm{dBm}$ is shown in Figure 4. The mean values for each distance as well as the calculated values using FSPL equation are shown in Figure 5.

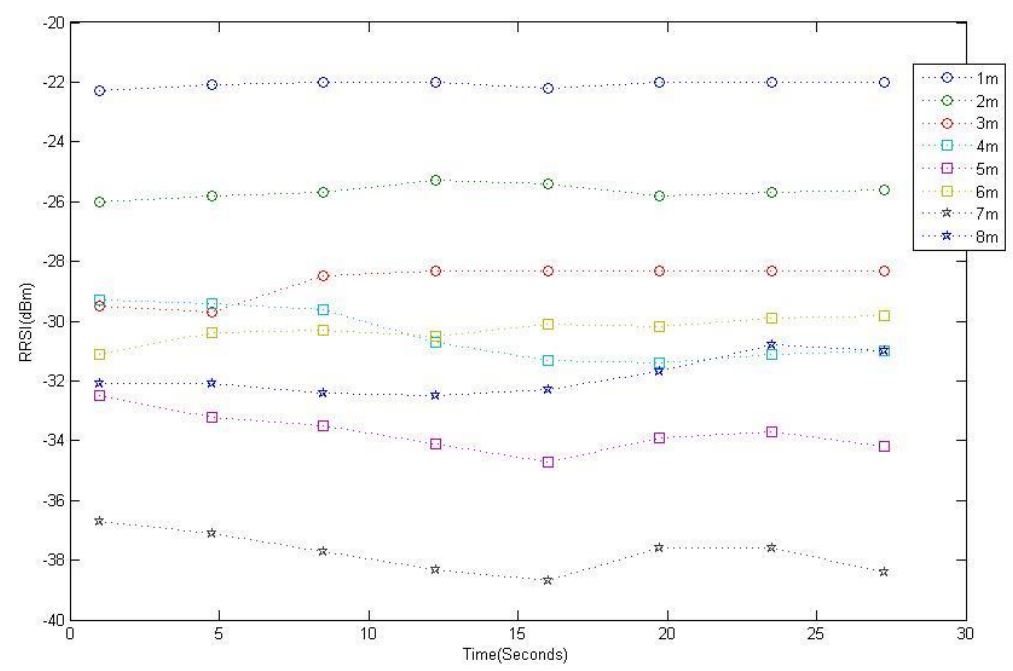

Figure 4. RSSI Value (Indoor)

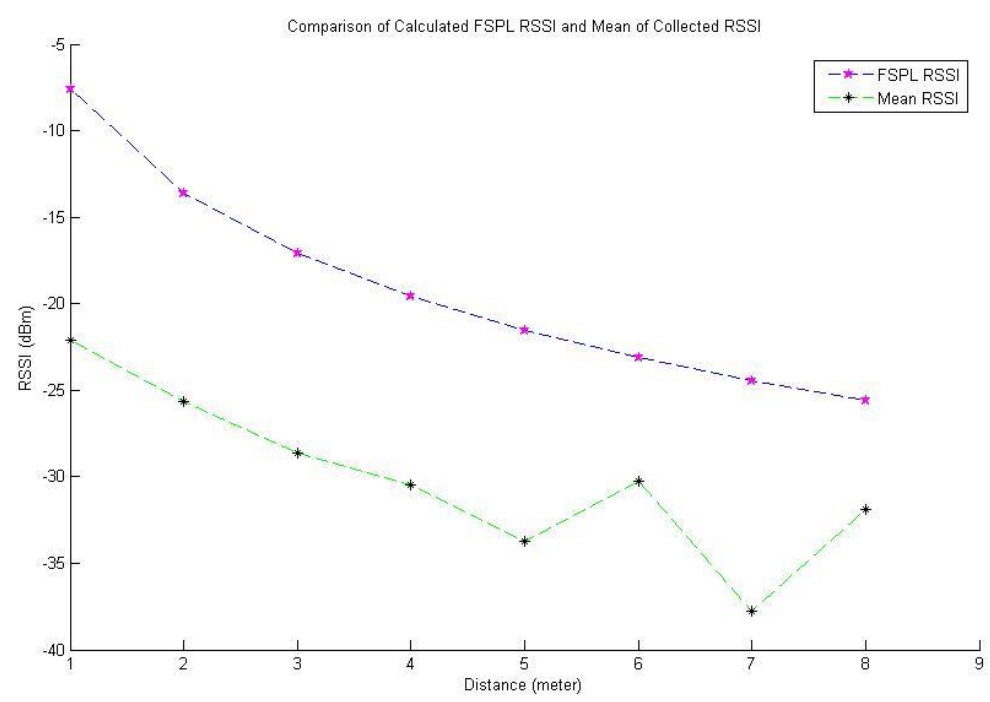

Figure 5. Comparison of Calculated RSSI and Measured RSSI vs Distance within Indoor Environment

The best fit linear equation is shown in Figure 6. The derived equation can be used to estimate other distance values based on received signal strength indication. A comparison between FSPL RSSI and Mean measured values vs $\log _{10}$ (Distance) is shown in Figure 7. The mean measured values, FSPL values, the differences between measured and FSPL values, the variances and standard deviations are enumerated in Table 1. Based on the values listed inTable 1, the average difference between measured values and FSPL values is $10.99463 \mathrm{~dB}$. 


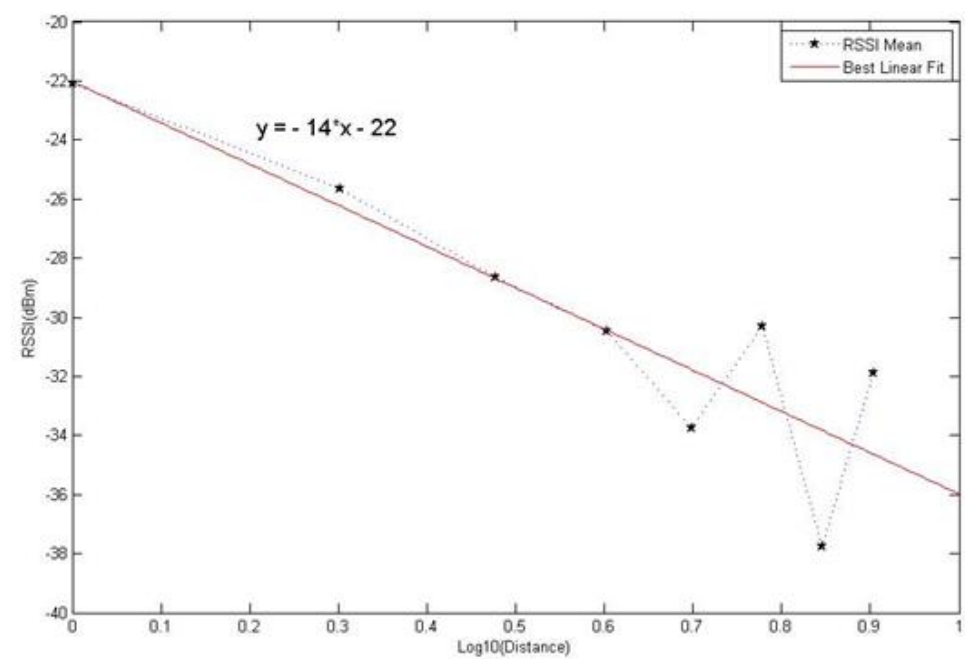

Figure 6. Plot of Average Measured Values of RSSI within an Enclosed Environment

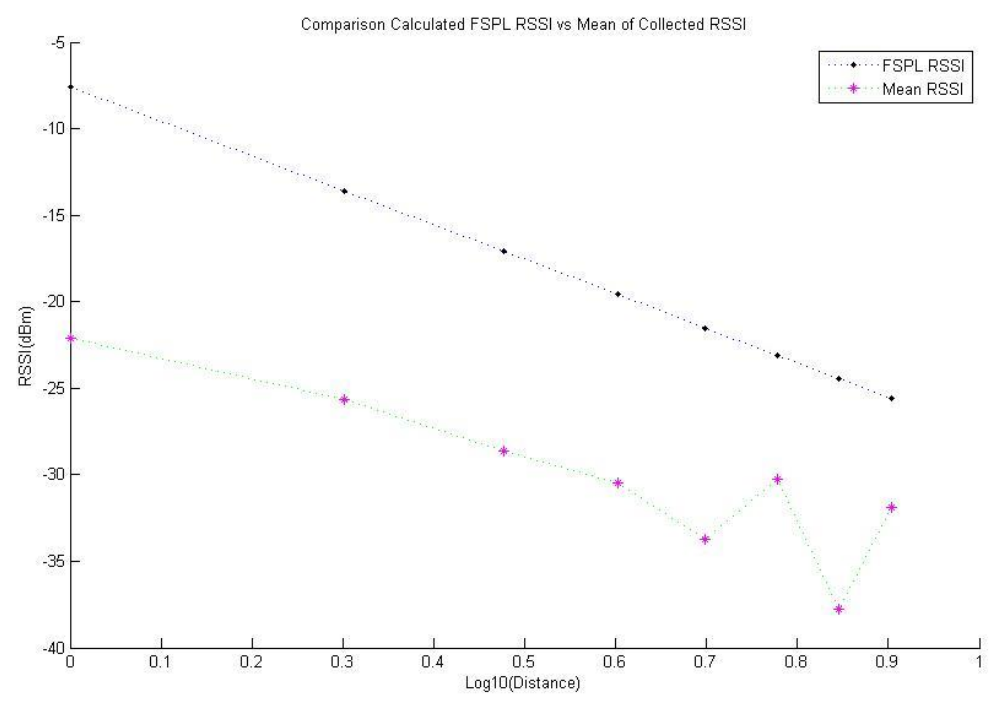

Figure 7. Comparison of Calculated FSPL RSSI and Actual RSSI vs $\log _{10}$ (Distance) within Indoor Environment 
Table 1. The Comparison between the Values of RSSI within an Enclosed Environment with FSPL Estimations

\begin{tabular}{|c|c|c|c|l|l|}
\hline Distance $(\mathrm{m})$ & $\begin{array}{c}\text { Mean } \\
\text { Measured }\end{array}$ & FSPL & Difference & Variance & $\begin{array}{c}\text { Standard } \\
\text { Deviation }\end{array}$ \\
\hline 1 & -22.075 & -7.553 & 14.522 & 0.013571429 & 0.116496475 \\
\hline 2 & -25.6625 & -13.57 & 12.0925 & 0.05125 & 0.226384628 \\
\hline 3 & -28.65 & -17.1 & 11.55 & 0.351428571 & 0.592814112 \\
\hline 4 & -30.475 & -19.59 & 10.885 & 0.793571429 & 0.890826262 \\
\hline 5 & -33.725 & -21.53 & 12.195 & 0.453571429 & 0.673477118 \\
\hline 6 & -30.2875 & -23.12 & 7.1675 & 0.164107143 & 0.405101398 \\
\hline 7 & -37.7625 & -24.46 & 13.3025 & 0.456964286 & 0.675991336 \\
\hline 8 & -31.8625 & -25.62 & 6.2425 & 0.414107143 & 0.643511572 \\
\hline
\end{tabular}

\section{Derivation of RSSI and Distance Correlation}

From the mean values as shown in Figure 6, the best fit linear regression was applied to produce equation 14. The equation can be used to calculate distances based on the RSSI quantities acquired in an interior environment. An equation was generated using simple fitting tool of MATLAB when applied on the linear curve amid the RSSI and $\log _{10}$ (Distance). Related relationship between the RSSI and $\log _{10}$ (Distance) is labeled as Equation 14:

$$
y=-14 x-22
$$

$y$ represents the RSSI and $x$ represents $\log _{10}$ (Distance). From this equation the distance within indoor environment would be:

$$
D=10^{\left(\frac{y+22}{-14}\right)}
$$

\section{Conclusion}

As a conclusion, the performance evaluation within an enclosed environment was acquired. From the results obtained, the difference between measured and FSPL values for indoor environment is $0.99463 \mathrm{~dB}$. It can be concluded that the result is not too much deviated and could be possible to use as approximation algorithm under the ideal condition for determining the GSM1800 range. A current experiment is in progress in outdoor environment as well as in clean room environment as an improvement and validation of the current model.

\section{Acknowledgements}

The presented investigation outcomes are segment of the deliverables for the funded research by Malaysian Ministry of Higher Education and Research Management Centre of the International Islamic University Malaysia (IIUM). Authors acknowledge the support and express utmost gratitude for the financial provision. 


\section{References}

[1] I.F. Akyildiz, W. Su, Y. Sankarasubramamiam, E. Cayirci, Wireless Sensor Networks: A Survey, Elsivier Science B.V Journal of Computer Networks, 393-422 (2002).

[2] Z. Fang, Z. Zhao, P. Guo, et al., Analysis of Distance Measurement Based on RSSI, Chinese Journal of Sensors and Actuators, Vol. 20, No. 11, 2007, pp. 25262530.

[3] Adewumi, Omotayo G., Karim Djouani, and Anish M. Kurien. RSSI based indoor and outdoor distance estimation for localization in WSN, Industrial Technology (ICIT), 2013 IEEE International Conference on. IEEE, 2013.

[4] Kumar, Praveen, Lohith Reddy, and Shirshu Varma. Distance measurement and error estimation scheme for RSSI based localization in Wireless Sensor Networks, Wireless Communication and Sensor Networks (WCSN), 2009 Fifth IEEE Conference on. IEEE, 2009.

[5] Feng, Chen, et al. Received-signal-strength-based indoor positioning using compressive sensing, IEEE Transactions on Mobile Computing 11.12 (2012): 1983-1993.

[6] Zickler, Stefan, and Manuela Veloso. RSS-based relative localization and tethering for moving robots in unknown environments, Robotics and Automation (ICRA), 2010 IEEE International Conference on. IEEE, (2010).

[7] Wang, Jiing-Yi, et al. High-precision RSSI-based indoor localization using a transmission power adjustment strategy for wireless sensor networks, High Performance Computing and Communication \& 2012 IEEE 9th International Conference on Embedded Software and Systems (HPCC-ICESS), 2012 IEEE 14th International Conference on. IEEE, (2012).

[8] Ranjeet Tomar \& G.S. Tomar, "Performance Evaluation Techniques for Mobile Communication Systems", IEEE International Conference CICSYN 2009, pp 511-515, July 2009.

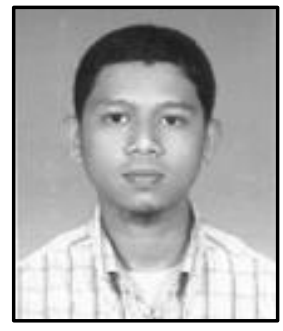

Muhamad Jamil bin Jakpar, currently study for Master of Science in Communication Engineering in International Islamic University of Malaysia (IIUM). He completed his Bachelor degree in Electrical and Computer Engineering (Communication) from International Islamic University of Malaysia IIUM.

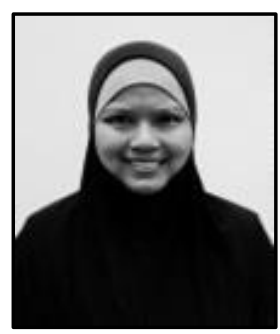

Nor Farahidah Za'bah, a former graduate of the Universiti Tenaga Nasional in Kajang, Selangor started her career as a Process Engineer in Silterra Malaysia, Kulim Hi Tech Park, Kedah where she provided process-engineering support to the CMOS manufacturing team. In 2004, after receiving her MSc from Newcastle University, UK, she was appointed as a Lecturer at Universiti Tenaga Nasional. In 2007, she accepted an offer as a lecturer in International Islamic and a year later, she pursued her $\mathrm{PhD}$ in Newcastle University, UK. Her research was focusing on the fabrication of silicon nanowire using the top-down approach where she was exposed to work in a wafer fabrication and characterization facilities such as cleanrooms and using measurement tools such as AFM. She received her $\mathrm{PhD}$ in 2012 and currently, she is attached as an Assistant Professor to the Department of Electrical and Computer Engineering, International Islamic University Malaysia, Kuala Lumpur. 